\title{
Evaluation on Comprehensive Ability of Inspection and Testing Organization of Legal Person in Hebei Province - Taking Construction Engineering Field as an Example
}

\author{
Jiaxing $\mathrm{He}$ \\ School of HeBei, Science and Technology University, ShiJiazhuang 050018, China; \\ 172624004@qq.com
}

Keywords: Inspection and testing, construction engineering, comprehensive ability, entropy method, TOPSIS

\begin{abstract}
This paper starts from the field of construction engineering, discusses the comprehensive ability of inspection and testing institutions in Hebei Province, uses the entropy method to determine the weight of each part, and uses the TOPSIS method to sort out the comprehensive ability score of the construction engineering field of Hebei province enterprise legal person unit inspection and testing institution the evaluation model is constructed and the problems existing in the in it and analyzed according to the evaluation results.
\end{abstract}

\section{Introduction}

With the development of the times and social progress, people for the inspection and testing organizations continue to improve,the demand for inspection and testing activities attracted people's continued attention and recognition. Hebei Province is currently engaged in inspection and testing of 1642 institutions, involving construction of machinery, energy, chemicals, mining, metallurgy, water quality and other fields. Hebei Province, the field of construction inspection and testing totally 270, throughout the various regions of Hebei Province. This paper starts from the field of construction engineering, uses the entropy method to determine the weight of each part, and constructs the technical capability evaluation model of the construction engineering industry in Hebei province. Through the assessment of the comprehensive capacity of the inspection and testing institutions in Hebei Province, it will help us to better understand the technical capability, competitiveness and market potential of the inspection and testing organizations in the construction industry, and provide new ones for the reform and development of inspection and testing, with the existence of the problem, put forward suggestions for improvement.

2. Determination of Comprehensive Ability Evaluation Index of Inspection and Testing Organization of Construction Personnel in Hebei Province. There are 237 inspection and testing institutions in Hebei Province, which are located in 11 subordinate cities of Hebei Province, and provide support for inspection and testing activities in their respective regions. According to the information collected, this paper evaluates the comprehensive ability of inspection and testing of legal person units in Hebei Province from four aspects: basic condition, enterprise efficiency, scientific research ability and human resources. By selecting the influencing factors set, the firstLevel 1 index set down sub-42 secondary indicators. As shown in Table 1.

Table 1 Construction of evaluation index system

Level 1 indicators

Leve 2 indicators

\footnotetext{
All the number of instruments(V1)

More than 400,000 instruments (V2)

Number of imported equipment (V3)

Total value of total output value (V4)

400000 or more asset value (V5)

The total value of imported equipment
} 
Total area of the organization (V7)

Office area (V8)

Laboratory area (V9)

Constant temperature and humidity laboratory area (V10)

Dedicated outdoor laboratory area (V11)

Total value of fixed assets (V12)

Total number of units (V13)

Number of graduate students (V14)

Number of undergraduates (V15)

Number of junior college students (V16)

Human Resources (U2) Senior technical titles (V17)

Intermediate technical title (V18)

Junior technical title (V19)

Other technical titles (V20)

Number of managers (V21)

Social commission report income (V22)

Other reported income (V23)

Provincial project amount (V24)

Business Efficiency (U3) Revision of the standard system (V25)

Operating profit (V26)

Government subsidy income (V27)

Total funding for research projects (V28)

Number of Administrative Enforcement Reports（V29）

Number of social commissioned reports (V30)

Number of other reports (V31)

Product Standards (V32)

Method standard (V33)

National standard (V34)

Research Ability (U4)

Revision of industry standards (V35)

Revision of local standards (V36)

Participate in competency verification program (V37)

Participate in measurement review (V38)

Number of national certificates (V39)

Number of provincial certificates (V40)

Total research projects (V41)

Provincial project totals (V42)

\section{The entropy method is used to determine the weight of the index \\ 3.1 Indicators are quantified}

According to the survey data of Hebei province in 2015, combined with the four primary evaluation indexes of this article, there are 42 secondary evaluation indexes under the primary evaluation index, and the evaluation information matrix is obtained. Due to different indicators, the scale of measurement is not the same, the need for the evaluation of the same degree of quantification, data standardization: 
Positive indicators: $X i j=\frac{X i j-\min \{X j\}}{\max \{X j\}-\min \{X j\}}$, Negative indicators: $X i j=\frac{\max \{X j\}-X i j}{\max \{X j\}-\min \{X j\}}$

Based on the normalization of the positive and negative indicators, the proportion of the index of the jth indicator in the ith unit is calculated on the basis of this: $\quad Y i j=\frac{X i j}{\sum_{i=1}^{m} X i j}$.

We can get the decision matrix.

Thus, calculate the j-th indicator information entropy: $e j=-k \sum_{i=1}^{m}(\text { Yij } \times \operatorname{InYij})^{, \mathrm{k}}=\frac{1}{\mathrm{InM}}$.

\subsection{Determine the weight of the indicator.}

Before calculating the weight, we must first set the difference coefficient of $\mathrm{j}$ index ${ }^{X_{i j}}$,for a given $\mathrm{j}$, the index value of each scheme ${ }^{x_{i j}}$,The smaller the difference coefficient, the bigger $e_{j}$ is.When $e_{j}=e_{\max }=1, e_{\max }$ is the maximum entropy.From the comparing we can learn that,when the difference $x_{i j}$ is bigger, ${ }^{e_{j}}$ is smaller.Difference coefficient $g_{j}=1-e_{j}$, the bigger ${ }^{e_{j}}$ is,the more importance the indicators. The difference coefficient vector is $\mathrm{G}=\left(\mathrm{g}_{1}, g_{2}, g_{3} \cdots g_{m}\right)$.The index weight is $w_{j=} \frac{\boldsymbol{g}_{j}}{\sum_{j=1}^{n} g_{j}}$.In order to evaluate the comprehensive capacity of a unit, there are 42 indicators of the evaluation index system, the establishment of mathematical model: $u=\left\{u_{1}, u_{2}, u_{3} \cdots u_{42}\right\}$, each sample consists of 42 indicators of data representation $u_{i}=\left\{X_{i 1}, X_{i 2}, X_{i 3} \cdots X_{i m}\right\}$, The original data matrix of the evaluation system can be obtained $\mathrm{X}=\left(\mathrm{x}_{\mathrm{ij}}\right)_{m \times n}$, the data normalization matrix is $\mathrm{Y}=\left\{y_{i j}\right\}_{m \times n}$, The bigger the gap between $^{X_{i j}}$, The greater the role of the indicator in the comprehensive evaluation.Information entropy of $X_{j} e_{i s} e_{j}=-k \sum_{i=1}^{42} y_{i j} I_{i j}$, K related to the number of samples of the system.Item $\mathrm{j}$ indicators ${ }_{j}$, the information utility value depends on the information entropy of the indicator,The difference between ej and $1, d_{j}=1-e_{j}$. The weight of each index is estimated by the entropy method. Its essence is calculated by using the value coefficient of the index information. The higher the value coefficient is, the more important the evaluation is. The weight of the index is: $\sum_{i=1}^{w_{j}=\frac{d_{j}}{\sum_{i}^{m} d_{j}}}$. For the multi-layer structure evaluation system, according to the information entropy can be added, the use of the underlying structure of the index information utility value, according to the proportion of the corresponding structure to determine the weight ${ }^{w_{j}}$, The utility value of each type of indicators of the lower structure is summed, and the utility value of each kind of index is obtained $D_{k}, k=1,2, \ldots$, $\mathrm{k}$, So as to get the sum of all the utility values $\mathrm{D}=\sum_{i=1}^{k} D_{i}$, Corresponding to the weight of each subsystem $^{w_{k}=\frac{D_{k}}{D}}$, The index corresponds to the weight of the superstructure $w_{j}=\frac{d_{j}}{D}$.

\subsection{Entropy method for comprehensive evaluation of the steps.}

First, the index values are not dimensioned.Put the indicators $x_{i j}$ into no relative number of units ${ }_{i j}$, At the same time the size of the specification in the [0,1], the analysis of sampling linear non-dimensional method,tthen let the dimensionless data being transformed and normalized.Finally, 
calculate the entropy of the indicator ${ }^{e_{j}}$ and redundancy ${ }^{d_{j}}$. And further get each unit comprehensive evaluation score,

$$
\operatorname{Scord}_{\mathrm{i}}=\sum_{j=1}^{n}\left(w_{j} \times p_{i j}\right)
$$

4. TOPSIS method to determine the comprehensive evaluation of the sort.

4.1Application of TOPSIS Decision Method.The alternatives are sorted by calculating the distance between the alternatives and the optimal solution and the worst solution, and the evaluation of the relative merits of the alternatives is obtained. "Ideal solution" and "negative ideal solution" are two basic concepts of law. So that the value of the index attribute values are reached the most satisfactory solution for the ideal solution; the contrary, so that the various attributes of the indicators are the most satisfactory value of the solution is negative ideal solution. By comparing the options and the ideal solution and the ideal solution of the close degree, so the merits of the program to sort, and then select the optimal program.

4.2 TOPSIS decision-making method specific steps.(1)To standardize the process, the decision matrix will be standardized into a matrixY.Its elements pass $\mathrm{z}_{\mathrm{ij}}=\frac{f_{i j}}{\sqrt{\sum_{i=1}^{n} f_{i j}^{2}}}$ $\mathrm{i}=1,2,3 \ldots . . . \mathrm{n} ; \mathrm{j}=1,2,3 \ldots . . \mathrm{m}$ to get the solution.(2)Constructing a Normalized Weighted Decision Matrix Z,Its elements $Z_{i j}, Z_{i j}=W_{j} Z_{i j}$, the weight value $W_{i j}$ obtained by the entropy method.(3)Determine the ideal solution and the negative ideal solution.The larger the element value in decision matrix better. $Z^{+}=\left(Z_{1}^{+}, Z_{2}^{+} \ldots . . Z_{n}^{+}\right)=\left\{\max Z_{i j} \mid \mathrm{j}=1,2,3 \ldots \mathrm{n}\right\} ; Z^{-}=\left(Z_{1}^{-}, Z_{2}^{-} \ldots Z_{m}^{-}\right)=\left\{\min Z_{i j} \mid \mathrm{j}=1,2 \ldots \mathrm{m}\right\}$. (4)Ca lculate the distance from the scheme to the ideal solution and the negative ideal solution. $\left.^{\left.d_{i}^{*}=\sqrt{\sum_{i=1}^{n}\left(Z_{i j}\right.}-Z^{+}\right)^{2}},(\mathrm{i}=1,2 \ldots . \mathrm{n}),{ }^{d_{i}^{-}}=\sqrt{\sum_{\mathrm{i}=1}^{m}\left(Z_{i j}\right.}-Z^{-}\right)^{2},(\mathrm{j}=1,2 \ldots \mathrm{m})$.

Calculate the proximity of each scheme to the ideal solution. $\mathrm{C}_{\mathrm{i}}=\frac{d_{i}^{-}}{\left(d_{i}^{*}+d_{i}^{-}\right)}(\mathrm{I}=1,2,3 \ldots . . \mathrm{n})$ According to the order from big to small to determine the sort, the greater the better, otherwise the worse.

\section{Analysis on the Evaluation Result of the Inspection and Testing Institution of the Legal Person in the Construction Industry of Hebei Province.}

According to the entropy method and TOPSIS comprehensive evaluation, the factors that affect the comprehensive ability level of the legal person unit are: the scientific research ability, the enterprise efficiency, the infrastructure, the human resources, the weight is $47.49 \%, 26.54 \%, 19.31 \%$, $6.63 \%$.

From the evaluation results, the evaluation results from the highest 0.51 to the lowest 0.02 , can be seen in different regions between the different units of the test level and ability to vary. Through the analysis of TOPSIS results, 11 cities in Hebei Province can be divided into three categories: inspection and testing of outstanding units; inspection and testing of advanced units; inspection and testing of general units. Evaluation value of more than 0.5 in one, for the test and testing of 
outstanding units; evaluation value of 0.1 or more 54 , for the detection of advanced units; evaluation value of 0.02 or more for the inspection and testing of general units. Based on the distribution of the evaluation values, the comprehensive distribution of inspection and testing institutions is uneven.

In the above comprehensive evaluation problem, it is clear that no unit has the best performance on all the indicators, so it is necessary to weigh the difference between each unit index in the evaluation. Inspection and testing of outstanding units in scientific research capacity and business efficiency accounted for significant advantages, inspection and testing units in the scientific research and innovation capacity, business efficiency and high-level personnel training need to improve.

\section{Summary}

In this paper, the entropy method and TOPSIS method are used to evaluate the comprehensive capability of the inspection and testing institutions in the construction engineering field of Hebei Province, and the comprehensive evaluation results of the inspection and testing organizations in the corporate units of Hebei Province are obtained. Analysis of the characteristics of the comprehensive ability of inspection and testing institutions and analysis of the main reasons for the existence of inspection and testing institutions. Through the comparative analysis of the evaluation results, we can further understand the relative advantages and relative disadvantages of the comprehensive ability of the inspection and testing institutions, so as to provide the decision-making basis for improving the overall level of China's inspection and testing institutions.

\section{References}

[1].Xu xu, Yonggang Zhao.Based on the entropy method and fuzzy comprehensive evaluation method of green supply chain performance evaluation. JOURNAL OF SHANGHAI DIANJI UNIVERSITY. Vol. 15(2012) No. 4, p. 231-236.

[2].Hengbo Du, Yanfeng Xu. Based on the entropy value method of human resource outsourcing risk fuzzy comprehensive evaluation research. Company Management. Vol. 5(2011) No. 5.

[3].Chuanlei Wang, Binglin Xu. Testing services in our country development present situation, problems and countermeasures[J].Technology and market,2013.05:297-300.

[4].Zhongyi Gu, Detao LI, The quality inspection basis, China standard publishing house

[5].Zhenhua Chen: Status analysis and enhance of inspection and testing ability of Zhejiang Province (Master Degree, Hebei University of Technology, CHINA, 2007).

[6].Xiaowei Hu:The Study on Construction of Henan Province quality supervision inspection public technical service platform(Master Degree,Zhengzhou University,CHINA,2014)

[7]Zhang Meifang,Present situation and the development of third party testing institutions, The modern industrial economy and information technology, N010.2011. 